International Mathematical Forum, 1, 2006, no. 13, 629-651

\title{
FOURIER-FEYNMAN TRANSFORM \\ AND FIRST VARIATION OF CYLINDER \\ TYPE FUNCTIONS OVER WIENER \\ PATHS IN ABSTRACT WIENER SPACE
}

\author{
Dong Hyun Cho \\ Department of Mathematics, Kyonggi University, \\ Suwon 443-760, Korea, e-mail: j94385@kyonggi.ac.kr
}

\begin{abstract}
In this paper, we express the analytic Feynman integral of the first variation of cylinder type functions $F$ in terms of the analytic Feynman integral of the product of $F$ with a linear factor over Wiener paths in abstract Wiener space. And then, we derive the Fourier-Feynman transform for the product of cylinder type functions which define the functions in a Banach algebra introduced by Yoo, with $n$ linear factors.
\end{abstract}

Mathematics Subject Classification: 28C20

Keywords: classical Wiener space, cylinder type functions, first variation, Fourier-Feynman transform, Fresnel class, Wiener paths in abstract Wiener space

\section{Introduction and preliminaries}

Let $C_{0}[0, T]$ be the space of all continuous paths $x$ on $[0, T]$ with $x(0)=0$ which is known as the classical Wiener space. The concept of an $L_{1}$ analytic Fourier-Feynman transform for the functionals on this space was introduced by Brue in [1]. In [3], Cameron and Storvick introduced an $L_{2}$ analytic FourierFeynman transform, and in [10] Johnson and Skoug developed an $L_{p}$ analytic Fourier-Feynman transform theories for $1 \leq p \leq 2$ that extended the results in $[1,3]$ and gave various relationships between the $L_{1}$ and $L_{2}$ theories.

On the other hand, in [2], Cameron obtained the Wiener integral of the first variation of a functional $F$ in terms of the Wiener integral of the product $F$ with a linear factor. In [14], Park, Skoug and Storvick found the FourierFeynman transform of the product of a functional, with $n$ linear factors, from 
the Banach algebra $\mathcal{S}$ which was introduced by Cameron and Storvick in [4]. In [6], Chang, Song and Yoo expressed the analytic Feynman integral of the first variation of a functional $F$ in terms of the analytic Feynman integral of the product of $F$ with a linear factor on abstract Wiener space. And then, they derived the Fourier-Feynman transform for the product of a functional in the Fresnel class with $n$ linear factors.

In [7], Cho introduced a Banach algebra $\mathcal{F}\left(C_{0}(\mathbb{B}) ; u\right)$ which is equivalent to the Fresnel class and in [16], Yoo did the Banach algebra $S_{\mathbb{B}}^{\prime \prime}$ which corresponds to the Banach algebra $\mathcal{S}^{\prime \prime}$ introduced by Cameron and Storvick in [4]. And also, in [8], Cho extended the results in [6] to the functionals in $\mathcal{F}\left(C_{0}(\mathbb{B}) ; u\right)$ over Wiener paths in abstract Wiener space.

In this paper, with cylinder type functions which defines the functionals in $S_{\mathbb{B}}^{\prime \prime}$, we extend the results in [6] over Wiener paths in abstract Wiener space. Further, for this type of functions $F$, we express the analytic Feynman integral of the first variation of $F$ in terms of the analytic Feynman integral of the product of $F$ with a linear factor over Wiener paths in abstract Wiener space. And then, we derive the Fourier-Feynman transform of the product of $F$ with $n$ linear factors.

Let $(\mathcal{H}, \mathbb{B}, m)$ be an abstract Wiener space $([13])$. Let $\left\{e_{j}: j \geq 1\right\}$ be a complete orthonormal set in the real separable Hilbert space $\mathcal{H}$ such that $e_{j}$ 's are in $\mathbb{B}^{*}$, the dual space of real separable Banach space $\mathbb{B}$. For each $h \in \mathcal{H}$ and $y \in \mathbb{B}$, define the stochastic inner product $(h, y)^{\sim}$ by

$$
(h, y)^{\sim}= \begin{cases}\lim _{n \rightarrow \infty} \sum_{j=1}^{n}\left\langle h, e_{j}\right\rangle\left(y, e_{j}\right), & \text { if the limit exists } \\ 0, & \text { otherwise }\end{cases}
$$

where $(\cdot, \cdot)$ denotes the dual pairing between $\mathbb{B}$ and $\mathbb{B}^{*}([11])$. Note that for each $h(\neq 0)$ in $\mathcal{H},(h, \cdot)^{\sim}$ is a Gaussian random variable on $\mathbb{B}$ with mean zero, variance $|h|^{2}$; also $(h, y)^{\sim}$ is essentially independent of choice of the complete orthonormal set used in its definition and further, $(h, \lambda y)^{\sim}=(\lambda h, y)^{\sim}=\lambda(h, y)^{\sim}$ for all $\lambda \in \mathbb{R}$. It is well-known that if $\left\{h_{1}, h_{2}, \cdots, h_{n}\right\}$ is an orthogonal set in $\mathcal{H}$, then the random variables $\left(h_{j}, \cdot\right)^{\sim}$ 's are independent. Moreover, if both $h$ and $y$ are in $\mathcal{H}$, then $(h, y)^{\sim}=\langle h, y\rangle$ where $\langle\cdot, \cdot\rangle$ denotes the inner product of $h$ and $y$.

Let $C_{0}(\mathbb{B})$ denote the set of all continuous functions on $[0, T]$ into $\mathbb{B}$ which vanish at 0 . Then $C_{0}(\mathbb{B})$ is a real separable Banach space with the norm $\|x\|_{C_{0}(\mathbb{R})} \equiv \sup _{0 \leq t \leq T}\|x(t)\|_{\mathbb{B}}$. The minimal $\sigma$-field making the mapping $x \rightarrow$ $x(t)$ measurable is $\mathcal{B}\left(C_{0}(\mathbb{B})\right)$, the Borel $\sigma$-field on $C_{0}(\mathbb{B})$. Further, Brownian motion in $\mathbb{B}$ induces a probability measure $m_{\mathbb{B}}$ on $\left(C_{0}(\mathbb{B}), \mathcal{B}\left(C_{0}(\mathbb{B})\right)\right)$ which is mean-zero Gaussian([15]). We will find a concrete form of $m_{\mathbb{B}}$. Let $\vec{t}=$ $\left(t_{1}, t_{2}, \cdots, t_{k}\right)$ be given with $0=t_{0}<t_{1}<t_{2}<\cdots<t_{k} \leq T$. Let $T_{\vec{t}}: \mathbb{B}^{k} \rightarrow \mathbb{B}^{k}$ 
be given by

$$
\begin{aligned}
& T_{\vec{t}}\left(x_{1}, x_{2}, \cdots, x_{k}\right) \\
= & \left(\sqrt{t_{1}-t_{0}} x_{1}, \sqrt{t_{1}-t_{0}} x_{1}+\sqrt{t_{2}-t_{1}} x_{2}, \cdots, \sum_{j=1}^{k} \sqrt{t_{j}-t_{j-1}} x_{j}\right) .
\end{aligned}
$$

We define a set function $\nu_{\vec{t}}$ on $\mathcal{B}\left(\mathbb{B}^{k}\right)$ by

$$
\nu_{\vec{t}}(B)=\left(\prod_{1}^{k} m\right)\left(T_{\vec{t}}^{-1}(B)\right)
$$

for $B \in \mathcal{B}\left(\mathbb{B}^{k}\right)$. Then $\nu_{\vec{t}}$ is a Borel measure. Let $f_{\vec{t}}: C_{0}(\mathbb{B}) \rightarrow \mathbb{B}^{k}$ be the function defined by

$$
f_{\vec{t}}(x)=\left(x\left(t_{1}\right), x\left(t_{2}\right), \cdots, x\left(t_{k}\right)\right) .
$$

For Borel subsets $B_{1}, B_{2}, \cdots, B_{k}$ of $\mathbb{B}, f_{\vec{t}}^{-1}\left(\prod_{j=1}^{k} B_{j}\right)$ is called the $I$-set with respect to $B_{1}, B_{2}, \cdots, B_{k}$. Then the collection $\mathcal{I}$ of all $I$-sets is a semi-algebra. We define a set function $m_{\mathbb{B}}$ on $\mathcal{I}$ by

$$
m_{\mathbb{B}}\left(f_{\vec{t}}^{-1}\left(\prod_{j=1}^{k} B_{j}\right)\right)=\nu_{\vec{t}}\left(\prod_{j=1}^{k} B_{j}\right) .
$$

Then $m_{\mathbb{B}}$ is well-defined and countably additive on $\mathcal{I}$. Using Carathéodory extension process, we have a Borel measure $m_{\mathbb{R}}$ on $\mathcal{B}\left(C_{0}(\mathbb{B})\right)$.

Now, we introduce Wiener integration theorem without proof. For the proof, see [15].

Theorem 1.1 (Wiener integration theorem) Let $\vec{t}=\left(t_{1}, t_{2}, \cdots, t_{k}\right)$ be given with $0=t_{0}<t_{1}<t_{2}<\cdots<t_{k} \leq T$ and let $f: \mathbb{B}^{k} \rightarrow \mathbb{C}$ be a Borel measurable function. Then

$$
\begin{aligned}
& \int_{C_{0}(\mathbb{B})} f\left(x\left(t_{1}\right), x\left(t_{2}\right), \cdots, x\left(t_{k}\right)\right) d m_{\mathbb{B}}(x) \\
\stackrel{*}{=} & \int_{\mathbb{B}^{k}}\left(f \circ T_{\vec{t}}\right)\left(x_{1}, x_{2}, \cdots, x_{k}\right) d\left(\prod_{j=1}^{k} m\right)\left(x_{1}, x_{2}, \cdots, x_{k}\right),
\end{aligned}
$$

where by $\stackrel{*}{=}$ we mean that if either side exists, then both sides exist and they are equal.

A subset $E$ of $C_{0}(\mathbb{B})$ is called a scale-invariant null set if $m_{\mathbb{E}}(\lambda E)=0$ for any $\lambda>0$. A property is said to hold scale-invariant almost everywhere (in 
abbreviation, $s$-a.e.) if it holds except for a scale-invariant null set. Let $F$ be defined on $C_{0}(\mathbb{B})$. Suppose that $E\left[F\left(\lambda^{-\frac{1}{2}} \cdot\right)\right]$ exists for any $\lambda>0$ and it has the analytic extension $J_{\lambda}^{*}(F)$ on $\mathbb{C}_{+} \equiv\{\lambda \in \mathbb{C}: \operatorname{Re} \lambda>0\}$. Then we call $J_{\lambda}^{*}(F)$ the analytic Wiener integral of $F$ with parameter $\lambda$ and it is denoted by

$$
E^{a n w_{\lambda}}[F]=J_{\lambda}^{*}(F)
$$

Moreover, for a non-zero real $q$, if $E^{a n w_{\lambda}}[F]$ has a limit as $\lambda$ approaches to $-i q$ through $\mathbb{C}_{+}$, then it is called the analytic Feynman integral of $F$ with parameter $q$ and denoted by

$$
\int_{C_{0}(\mathbb{B})}^{a n f_{q}} F(x) d m_{\mathbb{B}}(x)=E^{a n f_{q}}[F]=\lim _{\lambda \rightarrow-i q} E^{a n w_{\lambda}}[F] .
$$

\section{Feynman integrals of variations over Wiener paths in abstract Wiener space}

In this section, we define the first variation of functionals over Wiener paths in abstract Wiener space. And then, we investigate its properties.

Definition 2.1 Let $F$ be a functional defined on $C_{0}(\mathbb{B})$ and let $w \in C_{0}(\mathbb{B})$. The derivative

$$
\left.\frac{\partial}{\partial t} F(x+t w)\right|_{t=0}
$$

for $x \in C_{0}(\mathbb{B})$ if it exists, is called the first variation of $F$ at $x$ in the direction of $w$ and denoted by

$$
\delta_{w} F(x)=\left.\frac{\partial}{\partial t} F(x+t w)\right|_{t=0}
$$

Suppose that, for some $\epsilon>0, \delta_{w} F(x+t w)$ exists for $|t|<\epsilon$. Then we have

$$
\begin{aligned}
\delta_{w} F(x+t w) & =\left.\frac{\partial}{\partial \lambda} F(x+t w+\lambda w)\right|_{\lambda=0} \\
& =\left.\frac{\partial}{\partial \mu} F(x+\mu w)\right|_{\mu=t}=\frac{\partial}{\partial t} F(x+t w) .
\end{aligned}
$$

Let $\mathcal{H}$ be an infinite dimensional separable real Hilbert space and let $\Delta_{n}=$ $\left\{\left(s_{1}, \cdots, s_{n}\right): 0=s_{0}<s_{1}<\cdots<s_{n} \leq T\right\}$ for $n \in \mathbb{N}$. For

$$
\vec{s}_{n}=\left(s_{1}, \cdots, s_{n}\right) \in \Delta_{n} \text { and } \vec{h}_{n}=\left(h_{1}, \cdots, h_{n}\right) \in \mathcal{H}^{n}
$$


let

$$
F_{\vec{s}_{n}, \vec{h}_{n}}(x)=\exp \left\{i \sum_{j=1}^{n}\left(h_{j}, x\left(s_{j}\right)\right)^{\sim}\right\}
$$

for $x \in C_{0}(\mathbb{B})$. Further, for $w \in C_{0}(\mathbb{B})$ with $w\left(s_{j}\right) \in \mathcal{H}(j=1,2, \cdots, n)$, let

$$
F_{w, \vec{s}_{n}, \vec{h}_{n}}(x)=F_{\vec{s}_{n}, \vec{h}_{n}}(x) \sum_{j=1}^{n}\left(w\left(s_{j}\right), x\left(s_{j}\right)\right)^{\sim}
$$

for $x \in C_{0}(\mathbb{B})$ where $F_{\vec{s}_{n}, \vec{h}_{n}}$ is given by (3).

Let $\mathcal{M}_{n}^{\prime \prime}=\mathcal{M}_{n}^{\prime \prime}\left(\Delta_{n} \times \mathcal{H}^{n}\right)$ be the class of all complex Borel measures on $\Delta_{n} \times \mathcal{H}^{n}$ and let $\left\|\mu_{n}\right\|=\operatorname{var} \mu_{n}$, the total variation of $\mu_{n}$ in $\mathcal{M}_{n}^{\prime \prime}$. Let $S_{n, \mathbb{B}}^{\prime \prime}=$ $S_{n, \mathbb{B}}^{\prime \prime}\left(\Delta_{n} \times \mathcal{H}^{n}\right)$ be the space of functionals of the form

$$
F_{n}(x)=\int_{\Delta_{n} \times \mathcal{H}^{n}} F_{\vec{s}_{n}, \vec{h}_{n}}(x) d \mu_{F_{n}}\left(\vec{s}_{n}, \vec{h}_{n}\right)
$$

for $s$-a.e. $x \in C_{0}(\mathbb{B})$, where $\mu_{F_{n}}$ is in the class $\mathcal{M}_{n}^{\prime \prime}$ and $F_{\vec{s}_{n}, \vec{h}_{n}}$ is given by (3). Here we take $\left\|F_{n}\right\|_{n}^{\prime \prime}=\inf \left\{\left\|\mu_{F_{n}}\right\|\right\}$, where the infimum is taken over all $\mu_{F_{n}}$ 's so that $F_{n}$ and $\mu_{F_{n}}$ are related by (5). Let $\mathcal{M}^{\prime \prime}=\mathcal{M}^{\prime \prime}\left(\sum \Delta_{n} \times \mathcal{H}^{n}\right)$ be the class of all sequences $\left\{\mu_{n}\right\}$ of measures such that each $\mu_{n}$ is in $\mathcal{M}_{n}^{\prime \prime}$ with $\sum_{n=1}^{\infty}\left\|\mu_{n}\right\|<\infty$. Let $S_{\mathbb{B}}^{\prime \prime}=S_{\mathbb{B}}^{\prime \prime}\left(\sum \Delta_{n} \times \mathcal{H}^{n}\right)$ be the space of functions on $C_{0}(\mathbb{B})$ of the form

$$
F(x)=\sum_{n=1}^{\infty} F_{n}(x)
$$

where each $F_{n}$ is in $S_{n, \mathbb{B}}^{\prime \prime}$ with $\sum_{n=1}^{\infty}\left\|F_{n}\right\|_{n}^{\prime \prime}<\infty$. The norm of $F$ is defined by $\|F\|^{\prime \prime}=\inf \left\{\sum_{n=1}^{\infty}\left\|F_{n}\right\|_{n}^{\prime \prime}\right\}$, where the infimum is taken over all representations of $F$ given by (6).

Now, we introduce a useful integral which appears in the proofs of several results. The proof follows from the fact that $(h, \cdot)^{\sim}$ is normally distributed with mean 0 and variance $|h|^{2}$ if $h \neq 0$.

Lemma 2.2 Let $(\mathcal{H}, \mathbb{B}, m)$ be an abstract Wiener space and let $h \in \mathcal{H}$. Suppose that $\alpha$ is pure imaginary or real. Then we have

$$
\int_{\mathbb{B}} \exp \left\{\alpha\left(h, x_{1}\right)^{\sim}\right\} d m\left(x_{1}\right)=\exp \left\{\frac{\alpha^{2}|h|^{2}}{2}\right\} .
$$

Lemma 2.3 Let $F_{\vec{s}_{n}, \vec{h}_{n}}$ be given by (3) and let $w$ be in $C_{0}(\mathbb{B})$. Then $\delta_{w} F_{\vec{s}_{n}, \vec{h}_{n}}(x)$ exists and given by

$$
\delta_{w} F_{\vec{s}_{n}, \vec{h}_{n}}(x)=i \sum_{j=1}^{n}\left(h_{j}, w\left(s_{j}\right)\right)^{\sim} F_{\vec{s}_{n}, \vec{h}_{n}}(x)
$$

for $x$ in $C_{0}(\mathbb{B})$. 
Proof. By the definition of first variation, we have

$$
\begin{aligned}
\left.\frac{\partial}{\partial t} F_{\vec{s}_{n}, \vec{h}_{n}}(x+t w)\right|_{t=0} & =\left.\frac{\partial}{\partial t} \exp \left\{i \sum_{j=1}^{n}\left(h_{j}, x\left(s_{j}\right)+t w\left(s_{j}\right)\right)^{\sim}\right\}\right|_{t=0} \\
& =i \sum_{j=1}^{n}\left(h_{j}, w\left(s_{j}\right)\right)^{\sim} F_{\vec{s}_{n}, \vec{h}_{n}}(x)
\end{aligned}
$$

for $x$ in $C_{0}(\mathbb{B})$, which is the desired result.

Theorem 2.4 Let $F_{n}$ be given by (5) and let $w \in C_{0}(\mathbb{B})$. Suppose that

$$
\int_{\Delta_{n} \times \mathcal{H}^{n}} \mid \sum_{j=1}^{n}\left(h_{j}, w\left(s_{j}\right)\right) \sim d\left\|\mu_{F_{n}}\right\|\left(\vec{s}_{n}, \vec{h}_{n}\right)<\infty
$$

where $\vec{s}_{n}$ and $\vec{h}_{n}$ are given by (2). Then $\delta_{w} F_{n}(x)$ exists for s-a.e. $x \in C_{0}(\mathbb{B})$ and given by

$$
\delta_{w} F_{n}(x)=\int_{\Delta_{n} \times \mathcal{H}^{n}} \delta_{w} F_{\vec{s}_{n}, \vec{h}_{n}}(x) d \mu_{F_{n}}\left(\vec{s}_{n}, \vec{h}_{n}\right)
$$

where $\delta_{w} F_{\vec{s}_{n}, \vec{h}_{n}}$ is given by ( 7$)$.

Proof. For s-a.e. $x \in C_{0}(\mathbb{B})$, we have

$$
\begin{aligned}
\delta_{w} F_{n}(x) & =\left.\frac{\partial}{\partial t} \int_{\Delta_{n} \times \mathcal{H}^{n}} F_{\vec{s}_{n}, \vec{h}_{n}}(x+t w) d \mu_{F_{n}}\left(\vec{s}_{n}, \vec{h}_{n}\right)\right|_{t=0} \\
& =\left.\int_{\Delta_{n} \times \mathcal{H}^{n}} \frac{\partial}{\partial t} F_{\vec{s}_{n}, \vec{h}_{n}}(x+t w)\right|_{t=0} d \mu_{F_{n}}\left(\vec{s}_{n}, \vec{h}_{n}\right) \\
& =\int_{\Delta_{n} \times \mathcal{H}^{n}} \delta_{w} F_{\vec{s}_{n}, \vec{h}_{n}}(x) d \mu_{F_{n}}\left(\vec{s}_{n}, \vec{h}_{n}\right)
\end{aligned}
$$

where the second equality follows from (8) and [9, Theorem 2.27]. Hence the result follows.

Theorem 2.5 Let $F$ be given by $(6)$ and let $w \in C_{0}(\mathbb{B})$. Suppose that

$$
\sum_{n=1}^{\infty} \int_{\Delta_{n} \times \mathcal{H}^{n}} \mid \sum_{j=1}^{n}\left(h_{j}, w\left(s_{j}\right)\right) \sim d\left\|\mu_{F_{n}}\right\|\left(\vec{s}_{n}, \vec{h}_{n}\right)<\infty
$$


FOURIER-FEYNMAN TRANSFORM AND FIRST VARIATION

where $\vec{s}_{n}$ and $\vec{h}_{n}$ are given by (2). Then $\delta_{w} F(x)$ exists for s-a.e. $x \in C_{0}(\mathbb{B})$ and it is given by

$$
\delta_{w} F(x)=\sum_{n=1}^{\infty} \delta_{w} F_{n}(x)
$$

where $\delta_{w} F_{n}$ is given by (9).

Proof. For $s$-a.e. $x$ in $C_{0}(\mathbb{B})$ and $t$ in $\mathbb{R}$, we have

$$
\begin{aligned}
& \sum_{n=1}^{\infty}\left|\delta_{w} F_{n}(x+t w)\right| \\
= & \sum_{n=1}^{\infty}\left|\int_{\Delta_{n} \times \mathcal{H}^{n}} i \sum_{j=1}^{n}\left(h_{j}, w\left(s_{j}\right)\right)^{\sim} F_{\vec{s}_{n}, \vec{h}_{n}}(x+t w) d \mu_{F_{n}}\left(\vec{s}_{n}, \vec{h}_{n}\right)\right| \\
\leq & \sum_{n=1}^{\infty} \int_{\Delta_{n} \times \mathcal{H}^{n}}\left|\sum_{j=1}^{n}\left(h_{j}, w\left(s_{j}\right)\right)^{\sim}\right| d\left\|\mu_{F_{n}}\right\|\left(\vec{s}_{n}, \vec{h}_{n}\right)<\infty
\end{aligned}
$$

by (10), where $F_{\vec{s}_{n}, \vec{h}_{n}}$ is given by (3) so that we have

$$
\frac{\partial}{\partial t} F(x+t w)=\frac{\partial}{\partial t} \sum_{n=1}^{\infty} F_{n}(x+t w)=\sum_{n=1}^{\infty} \frac{\partial}{\partial t} F_{n}(x+t w)=\sum_{n=1}^{\infty} \delta_{w} F_{n}(x+t w)
$$

where the last equality follows form (1). Letting $t=0$, we have the result.

From now on, we will regard $s_{0}$ as 0 , unless otherwise specified.

Lemma 2.6 Let $F_{\vec{s}_{n}, \vec{h}_{n}}$ be given by (3). Then, for $\lambda>0$, we have

$$
\int_{C_{0}(\mathbb{B})} F_{\vec{s}_{n}, \vec{h}_{n}}\left(\lambda^{-\frac{1}{2}} x\right) d m_{\mathbb{B}}(x)=\exp \left\{-\frac{1}{2 \lambda} \sum_{l=1}^{n}\left(s_{l}-s_{l-1}\right)\left|\sum_{j=l}^{n} h_{j}\right|^{2}\right\} .
$$

Proof. By Theorem 1.1, for $\lambda>0$, we have

$$
\begin{aligned}
& \int_{C_{0}(\mathbb{R})} F_{\vec{s}_{n}, \vec{h}_{n}}\left(\lambda^{-\frac{1}{2}} x\right) d m_{\mathbb{B}}(x) \\
= & \int_{C_{0}(\mathbb{B})} \exp \left\{i \lambda^{-\frac{1}{2}} \sum_{j=1}^{n}\left(h_{j}, x\left(s_{j}\right)\right)^{\sim}\right\} d m_{\mathbb{B}}(x) \\
= & \int_{\mathbb{B}^{n}} \exp \left\{i \lambda^{-\frac{1}{2}} \sum_{j=1}^{n}\left(h_{j}, \sum_{l=1}^{j} \sqrt{s_{l}-s_{l-1}} x_{l}\right)^{\sim}\right\} d m^{n}(\vec{x}) \\
= & \int_{\mathbb{B}^{n}} \exp \left\{i \lambda^{-\frac{1}{2}} \sum_{l=1}^{n} \sqrt{s_{l}-s_{l-1}}\left(\sum_{j=l}^{n} h_{j}, x_{l}\right)^{\sim}\right\} d m^{n}(\vec{x})
\end{aligned}
$$


where $\vec{x}_{n}=\left(x_{1}, \cdots, x_{n}\right)$. Now, the result follows from Lemma 2.2.

Theorem 2.7 Let $F_{n}$ be given by (5) and let $w$ be in $C_{0}(\mathbb{B})$. Let $q \in \mathbb{R}-\{0\}$ and suppose that (8) holds. Then we have

$$
\begin{aligned}
& E^{a n f_{q}}\left[\delta_{w} F_{n}\right] \\
= & \int_{C_{0}(\mathbb{R})}^{a n f_{q}} \int_{\Delta_{n} \times \mathcal{H}^{n}} i \sum_{j=1}^{n}\left(h_{j}, w\left(s_{j}\right)\right)^{\sim} F_{\vec{s}_{n}, \vec{h}_{n}}(x) d \mu_{F_{n}}\left(\vec{s}_{n}, \vec{h}_{n}\right) d m_{\mathbb{B}}(x) \\
= & \int_{\Delta_{n} \times \mathcal{H}^{n}} i \sum_{j=1}^{n}\left(h_{j}, w\left(s_{j}\right)\right)^{\sim} \exp \left\{-\frac{i}{2 q} \sum_{l=1}^{n}\left(s_{l}-s_{l-1}\right)\left|\sum_{j=l}^{n} h_{j}\right|^{2}\right\} d \mu_{F_{n}}\left(\vec{s}_{n}, \vec{h}_{n}\right)
\end{aligned}
$$

where $F_{\vec{s}_{n}, \vec{h}_{n}}$ is given by (3).

Proof. By Theorems 1.1, 2.4 and Fubini's theorem, for $\lambda>0$, we have

$$
\begin{aligned}
& \int_{C_{0}(\mathbb{B})} \delta_{w} F_{n}\left(\lambda^{-\frac{1}{2}} x\right) d m_{\mathbb{B}}(x) \\
= & \int_{\Delta_{n} \times \mathcal{H}^{n}} i \sum_{j=1}^{n}\left(h_{j}, w\left(s_{j}\right)\right)^{\sim} \int_{C_{0}(\mathbb{B})} F_{\vec{s}_{n}, \vec{h}_{n}}\left(\lambda^{-\frac{1}{2}} x\right) d m_{\mathbb{B}}(x) d \mu_{F_{n}}\left(\vec{s}_{n}, \vec{h}_{n}\right) \\
= & \int_{\Delta_{n} \times \mathcal{H}^{n}} i \sum_{j=1}^{n}\left(h_{j}, w\left(s_{j}\right)\right)^{\sim} \exp \left\{-\frac{1}{2 \lambda} \sum_{l=1}^{n}\left(s_{l}-s_{l-1}\right)\left|\sum_{j=l}^{n} h_{j}\right|^{2}\right\} d \mu_{F_{n}}\left(\vec{s}_{n}, \vec{h}_{n}\right)
\end{aligned}
$$

where the last equality follows from Lemma 2.6. Now, the result follows from Morera's and the dominated convergence theorems.

Theorem 2.8 Let $F$ be given by $(6)$ and let $w \in C_{0}(\mathbb{B})$. Let $q \in \mathbb{R}-\{0\}$ and suppose that (10) holds. Then we have

$$
E^{a n f_{q}}\left[\delta_{w} F\right]=\sum_{n=1}^{\infty} E^{a n f_{q}}\left[\delta_{w} F_{n}\right]
$$

where $E^{a n f_{q}}\left[\delta_{w} F_{n}\right]$ is given as in Theorem 2.7.

Proof. By Theorem 2.4 and (10), for $\lambda>0$, we have

$$
\begin{aligned}
& \sum_{n=1}^{\infty} \int_{C_{0}(\mathbb{B})}\left|\delta_{w} F_{n}\left(\lambda^{-\frac{1}{2}} x\right)\right| d m_{\mathbb{B}}(x) \\
= & \sum_{n=1}^{\infty} \int_{C_{0}(\mathbb{B})}\left|\int_{\Delta_{n} \times \mathcal{H}^{n}} i \sum_{j=1}^{n}\left(h_{j}, w\left(s_{j}\right)\right)^{\sim} F_{\vec{s}_{n}, \vec{h}_{n}}\left(\lambda^{-\frac{1}{2}} x\right) d \mu_{F_{n}}\left(\vec{s}_{n}, \vec{h}_{n}\right)\right| d m_{\mathbb{B}}(x) \\
\leq & \sum_{n=1}^{\infty} \int_{\Delta_{n} \times \mathcal{H}^{n}}\left|\sum_{j=1}^{n}\left(h_{j}, w\left(s_{j}\right)\right)^{\sim}\right| d\left\|\mu_{F_{n}}\right\|\left(\vec{s}_{n}, \vec{h}_{n}\right)<\infty
\end{aligned}
$$


FOURIER-FEYNMAN TRANSFORM AND FIRST VARIATION

so that we have the result by Theorem 2.7, Morera's and the dominated convergence theorems.

Theorem 2.9 Let $w \in C_{0}(\mathbb{B})$ and let $F_{\vec{s}_{n}, \vec{h}_{n}}, F_{w, \vec{s}_{n}, \vec{h}_{n}}$ be given by (3), (4), respectively. Suppose that $w\left(s_{j}\right) \in \mathcal{H}$ for $j=1, \cdots, n$. Then we have the following integral formula

$$
\begin{aligned}
& \int_{C_{0}(\mathbb{B})} F_{w, \vec{s}_{n}, \vec{h}_{n}}(x) d m_{\mathbb{P}}(x) \\
= & i\left[\sum_{l=1}^{n}\left(s_{l}-s_{l-1}\right)\left\langle\sum_{j=l}^{n} h_{j}, \sum_{j=l}^{n} w\left(s_{j}\right)\right\rangle\right] \int_{C_{0}(\mathbb{B})} F_{\vec{s}_{n}, \vec{h}_{n}}(x) d m_{\mathbb{B}}(x)
\end{aligned}
$$

with the existences of the integrals of both sides of the equality.

Proof. By (11) with $\lambda=1$, we have

$$
\int_{C_{0}(\mathbb{B})} F_{\vec{s}_{n}, \vec{h}_{n}}(x) d m_{\mathbb{B}}(x)=\int_{\mathbb{B}^{n}} \exp \left\{i \sum_{l=1}^{n} \sqrt{s_{l}-s_{l-1}}\left(\sum_{j=l}^{n} h_{j}, x_{l}\right)^{\sim}\right\} d m^{n}(\vec{x})
$$

where $\vec{x}=\left(x_{1}, \cdots, x_{n}\right)$. By Cameron-Martin translation theorem $([12])$ and Theorem 1.1, we have, for $t \in \mathbb{R}$,

$$
\begin{aligned}
& \int_{C_{0}(\mathbb{B})} F_{\vec{s}_{n}, \vec{h}_{n}}(x) d m_{\mathbb{B}}(x) \\
= & \exp \left\{-\frac{1}{2} t^{2} \sum_{l=1}^{n}\left(s_{l}-s_{l-1}\right)\left|\sum_{j=l}^{n} w\left(s_{j}\right)\right|^{2}\right\} \int_{\mathbb{B}^{n}} \exp \left\{i \sum _ { l = 1 } ^ { n } \left(\sqrt{s_{l}-s_{l-1}} \sum_{j=l}^{n} h_{j},\right.\right. \\
& \left.\left.x_{l}+t \sqrt{s_{l}-s_{l-1}} \sum_{j=l}^{n} w\left(s_{j}\right)\right)^{\sim}\right\} \exp \left\{-t \sum_{l=1}^{n} \sqrt{s_{l}-s_{l-1}}\left(\sum_{j=l}^{n} w\left(s_{j}\right), x_{l}\right)^{\sim}\right\} \\
& d m^{n}(\vec{x}) \\
= & \exp \left\{-\frac{1}{2} t^{2} \sum_{l=1}^{n}\left(s_{l}-s_{l-1}\right)\left|\sum_{j=l}^{n} w\left(s_{j}\right)\right|^{2}\right\} \int_{\mathbb{B}^{n}} \exp \left\{i \sum _ { l = 1 } ^ { n } \sqrt { s _ { l } - s _ { l - 1 } } \left(\sum_{j=l}^{n} h_{j},\right.\right. \\
& \left.\left.x_{l}\right)^{\sim}\right\} \exp \left\{i t \sum_{l=1}^{n}\left(s_{l}-s_{l-1}\right)\left\langle\sum_{j=l}^{n} h_{j}, \sum_{j=l}^{n} w\left(s_{j}\right)\right\rangle\right\} \\
& \times \exp \left\{-t \sum_{l=1}^{n} \sqrt{s_{l}-s_{l-1}}\left(\sum_{j=l}^{n} w\left(s_{j}\right), x_{l}\right)^{\sim}\right\} d m^{n}(\vec{x}) \\
= & \exp \left\{-\frac{1}{2} t^{2} \sum_{l=1}^{n}\left(s_{l}-s_{l-1}\right)\left|\sum_{j=l}^{n} w\left(s_{j}\right)\right|^{2}\right\} \int_{C_{0}(\mathbb{B})}^{n} F_{\vec{s}_{n}, \vec{h}_{n}}(x) \exp \left\{-t \sum_{j=1}^{n}\left(w\left(s_{j}\right),\right.\right. \\
& \left.\left.x\left(s_{j}\right)\right)^{\sim}\right\} \exp \left\{i t \sum_{l=1}^{n}\left(s_{l}-s_{l-1}\right)\left\langle\sum_{j=l}^{n} h_{j}, \sum_{j=l}^{n} w\left(s_{j}\right)\right\rangle\right\} d m_{\mathbb{B}}(x) .
\end{aligned}
$$


Now, using similar method given as in the proof of Lemma 2.6, for $\alpha \in \mathbb{R}$, we have

$$
\begin{aligned}
& \int_{C_{0}(\mathbb{B})} \exp \left\{\alpha \sum_{j=1}^{n}\left(w\left(s_{j}\right), x\left(s_{j}\right)\right)^{\sim}\right\} d m_{\mathbb{B}}(x) \\
= & \exp \left\{\frac{\alpha^{2}}{2} \sum_{l=1}^{n}\left(s_{l}-s_{l-1}\right)\left|\sum_{j=l}^{n} w\left(s_{j}\right)\right|^{2}\right\}<\infty
\end{aligned}
$$

by Lemma 2.2. Take $\epsilon>0$, arbitrarily. Since $\sum_{j=1}^{n}\left|\left(w\left(s_{j}\right), x\left(s_{j}\right)\right)^{\sim}\right|<\sum_{j=1}^{n}$ $\exp \left\{\left|\left(w\left(s_{j}\right), x\left(s_{j}\right)\right)^{\sim}\right|\right\}$, both $\exp \left\{-t \sum_{j=1}^{n}\left(w\left(s_{j}\right), x\left(s_{j}\right)\right)^{\sim}\right\}$ and $\sum_{j=1}^{n}\left(w\left(s_{j}\right)\right.$, $\left.x\left(s_{j}\right)\right)^{\sim} \exp \left\{-t \sum_{j=1}^{n}\left(w\left(s_{j}\right), x\left(s_{j}\right)\right)^{\sim}\right\}$ are integrable functions of $x$ for $|t|<\epsilon$ by (14). Moreover, the integrals are independent of $t$. Differentiating both sides of (13) with respect to $t$, by [9, Theorem 2.27], we have

$$
\begin{aligned}
= & \int_{C_{0}(\mathbb{B})} F_{\vec{s}_{n}, \vec{h}_{n}}(x)\left[-t\left[\sum_{l=1}^{n}\left(s_{l}-s_{l-1}\right)\left|\sum_{j=l}^{n} w\left(s_{j}\right)\right|^{2}\right] \exp \left\{-\frac{1}{2} t^{2} \sum_{l=1}^{n}\left(s_{l}-s_{l-1}\right)\right.\right. \\
& \left.\times\left|\sum_{j=l}^{n} w\left(s_{j}\right)\right|^{2}\right\} \exp \left\{-t \sum_{j=1}^{n}\left(w\left(s_{j}\right), x\left(s_{j}\right)\right)^{\sim}\right\} \exp \left\{i t \sum _ { l = 1 } ^ { n } ( s _ { l } - s _ { l - 1 } ) \left\langle\sum_{j=l}^{n} h_{j},\right.\right. \\
& \left.\left.\sum_{j=l}^{n} w\left(s_{j}\right)\right\rangle\right\}+\exp \left\{-\frac{1}{2} t^{2} \sum_{l=1}^{n}\left(s_{l}-s_{l-1}\right)\left|\sum_{j=l}^{n} w\left(s_{j}\right)\right|^{2}\right\}\left[i \sum_{l=1}^{n}\left(s_{l}-s_{l-1}\right)\right. \\
& \left.\times\left\langle\sum_{j=l}^{n} h_{j}, \sum_{j=l}^{n} w\left(s_{j}\right)\right\rangle-\sum_{j=1}^{n}\left(w\left(s_{j}\right), x\left(s_{j}\right)\right)^{\sim}\right] \exp \left\{-t \sum_{j=1}^{n}\left(w\left(s_{j}\right), x\left(s_{j}\right)\right)^{\sim}\right\} \\
& \left.\times \exp \left\{i t \sum_{l=1}^{n}\left(s_{l}-s_{l-1}\right)\left\langle\sum_{j=l}^{n} h_{j}, \sum_{j=l}^{n} w\left(s_{j}\right)\right\rangle\right\}\right] d m_{\mathbb{B}}(x) .
\end{aligned}
$$

Letting $t=0$, we have the result.

Remark 2.10 For $\left(s_{1}, \cdots, s_{n}\right) \in \Delta_{n}$ and $\left(h_{1}, \cdots, h_{n}\right) \in \mathcal{H}^{n}$, we have

$$
\begin{aligned}
& \sum_{l=1}^{n}\left(s_{l}-s_{l-1}\right)\left\langle\sum_{j=l}^{n} h_{j}, \sum_{j=l}^{n} w\left(s_{j}\right)\right\rangle \\
= & \sum_{j=1}^{n} s_{j}\left\langle h_{j}, w\left(s_{j}\right)\right\rangle+\sum_{j=1}^{n-1} s_{j}\left\langle h_{j}, \sum_{l=j+1}^{n} w\left(s_{l}\right)\right\rangle+\sum_{j=1}^{n-1} s_{j}\left\langle\sum_{l=j+1}^{n} h_{l}, w\left(s_{j}\right)\right\rangle
\end{aligned}
$$

where $w \in C_{0}(\mathbb{B})$ with $w\left(s_{j}\right) \in \mathcal{H}$ for $j=1, \cdots, n$. Now, suppose that $\left\langle h_{j}, w\left(s_{l}\right)\right\rangle=0$ if $j \neq l$. Then (12) is reduced to the following equation:

$$
\int_{C_{0}(\mathbb{B})} F_{w, \vec{s}_{n}, \vec{h}_{n}}(x) d m_{\mathbb{B}}(x)=i\left[\sum_{j=1}^{n} s_{j}\left\langle h_{j}, w\left(s_{j}\right)\right\rangle\right] \int_{C_{0}(\mathbb{B})} F_{\vec{s}_{n}, \vec{h}_{n}}(x) d m_{\mathbb{B}}(x) .
$$


FOURIER-FEYNMAN TRANSFORM AND FIRST VARIATION

Let $0<u \leq T$. In particular, if $n=1$ and $s_{1}=u$, then we have

$$
\frac{1}{u} \int_{C_{0}(\mathbb{B})} F_{u, h}(x)(w(u), x(u))^{\sim} d m_{\mathbb{B}}(x)=\int_{C_{0}(\mathbb{B})} \delta_{w} F_{u, h}(x) d m_{\mathbb{B}}(x)
$$

with $h \equiv h_{1}$.

Theorem 2.11 Suppose that the assumptions given as in Theorem 2.9 hold. Then, for $q \in \mathbb{R}-\{0\}$, we have

$$
\begin{aligned}
& {\left[\sum_{l=1}^{n}\left(s_{l}-s_{l-1}\right)\left\langle\sum_{j=l}^{n} h_{j}, \sum_{j=l}^{n} w\left(s_{j}\right)\right\rangle\right] \int_{C_{0}(\mathbb{B})}^{a n f_{q}} F_{\vec{s}_{n}, \vec{h}_{n}}(x) d m_{\mathbb{B}}(x) } \\
= & -q \int_{C_{0}(\mathbb{R})}^{a n f_{q}} F_{w, \vec{s}_{n}, \vec{h}_{n}}(x) d m_{\mathbb{B}}(x)
\end{aligned}
$$

with the existences of the analytic Feynman integrals of the both sides of the equality.

Proof. For $\lambda>0$, with replacing $h_{j}$ by $\lambda^{-\frac{1}{2}} h_{j}$ in (12), we have

$$
\begin{aligned}
& i \lambda^{-\frac{1}{2}}\left[\sum_{l=1}^{n}\left(s_{l}-s_{l-1}\right)\left\langle\sum_{j=l}^{n} h_{j}, \sum_{j=l}^{n} w\left(s_{j}\right)\right\rangle\right] \int_{C_{0}(\mathbb{R})} F_{\vec{s}_{n}, \vec{h}_{n}}\left(\lambda^{-\frac{1}{2}} x\right) d m_{\mathbb{B}}(x) \\
= & \int_{C_{0}(\mathbb{R})} F_{\vec{s}_{n}, \vec{h}_{n}}\left(\lambda^{-\frac{1}{2}} x\right) \sum_{j=1}^{n}\left(w\left(s_{j}\right), x\left(s_{j}\right)\right)^{\sim} d m_{\mathbb{B}}(x) \\
= & \lambda^{\frac{1}{2}} \int_{C_{0}(\mathbb{R})} F_{w, \vec{s}_{n}, \vec{h}_{n}}\left(\lambda^{-\frac{1}{2}} x\right) d m_{\mathbb{B}}(x)
\end{aligned}
$$

by Theorem 2.9. Then we have

$$
\begin{aligned}
& \frac{i}{\lambda}\left[\sum_{l=1}^{n}\left(s_{l}-s_{l-1}\right)\left\langle\sum_{j=l}^{n} h_{j}, \sum_{j=l}^{n} w\left(s_{j}\right)\right\rangle\right] \int_{C_{0}(\mathbb{B})} F_{\vec{s}_{n}, \vec{h}_{n}}\left(\lambda^{-\frac{1}{2}} x\right) d m_{\mathbb{B}}(x) \\
= & \int_{C_{0}(\mathbb{R})} F_{w, \vec{s}_{n}, \vec{h}_{n}}\left(\lambda^{-\frac{1}{2}} x\right) d m_{\mathbb{B}}(x)
\end{aligned}
$$

and, letting $\lambda \rightarrow-i q$ through $\mathbb{C}_{+}$, by Morera's and the dominated convergence theorems, we have the result.

Remark 2.12 Let $q \in \mathbb{R}-\{0\}$. Under the assumptions given as in Remark 2.10, we have

$$
\frac{i u}{q} \int_{C_{0}(\mathbb{B})}^{a n f_{q}} \delta_{w} F_{u, h}(x) d m_{\mathbb{B}}(x)=\int_{C_{0}(\mathbb{B})}^{a n f_{q}} F_{u, h}(x)(w(u), x(u))^{\sim} d m_{\mathbb{B}}(x) .
$$


Lemma 2.13 Let $\mu_{F_{n}} \in \mathcal{M}_{n}^{\prime \prime}$ and let $w \in C_{0}(\mathbb{B})$ with $w(s) \in \mathcal{H}$ for all $s \in[0, T]$. Suppose that

$$
\int_{\Delta_{n} \times \mathcal{H}^{n}}\left|\sum_{l=1}^{n}\left(s_{l}-s_{l-1}\right)\left\langle\sum_{j=l}^{n} h_{j}, \sum_{j=l}^{n} w\left(s_{j}\right)\right\rangle\right| d\left\|\mu_{F_{n}}\right\|\left(\vec{s}_{n}, \vec{h}_{n}\right)<\infty
$$

where $\vec{s}_{n}$ and $\vec{h}_{n}$ are given by (2). Then we have

$$
\begin{aligned}
& \int_{C_{0}(\mathbb{B})} \int_{\Delta_{n} \times \mathcal{H}^{n}} F_{w, \vec{s}_{n}, \vec{h}_{n}}(x) d \mu_{F_{n}}\left(\vec{s}_{n}, \vec{h}_{n}\right) d m_{\mathbb{B}}(x) \\
= & i \int_{C_{0}(\mathbb{B})} \int_{\Delta_{n} \times \mathcal{H}^{n}} F_{\vec{s}_{n}, \vec{h}_{n}}(x) \sum_{l=1}^{n}\left[\left(s_{l}-s_{l-1}\right)\left\langle\sum_{j=l}^{n} h_{j}, \sum_{j=l}^{n} w\left(s_{j}\right)\right\rangle\right] \\
& d \mu_{F_{n}}\left(\vec{s}_{n}, \vec{h}_{n}\right) d m_{\mathbb{B}}(x)
\end{aligned}
$$

where $F_{\vec{s}_{n}, \vec{h}_{n}}, F_{w, \vec{s}_{n}, \vec{h}_{n}}$ are given by (3), (4), respectively.

Proof. Let $F_{n}$ be given by (5). Using similar method given as in the proof of Theorem 2.9, we have

$$
\begin{aligned}
& \int_{C_{0}(\mathbb{B})} F_{n}(x) d m_{\mathbb{B}}(x) \\
= & \int_{C_{0}(\mathbb{B})} \int_{\Delta_{n} \times \mathcal{H}^{n}} \exp \left\{-\frac{1}{2} t^{2} \sum_{l=1}^{n}\left(s_{l}-s_{l-1}\right)\left|\sum_{j=l}^{n} w\left(s_{j}\right)\right|^{2}\right\} \exp \left\{i t \sum_{l=1}^{n}\left(s_{l}-s_{l-1}\right)\right. \\
& \left.\times\left\langle\sum_{j=l}^{n} h_{j}, \sum_{j=l}^{n} w\left(s_{j}\right)\right\rangle\right\} \exp \left\{-t \sum_{j=1}^{n}\left(w\left(s_{j}\right), x\left(s_{j}\right)\right)^{\sim}\right\} F_{\vec{s}_{n}, \vec{h}_{n}}(x) d \mu_{F_{n}}\left(\vec{s}_{n}, \vec{h}_{n}\right) \\
& d m_{\mathbb{B}}(x)
\end{aligned}
$$

for $t \in \mathbb{R}$. Let $\|w\|_{C_{0}(\mathbb{B})}=\sup _{0 \leq s \leq T}\|w(s)\|_{\mathbb{B}}$. Then we have

$$
\begin{aligned}
& \sum_{l=1}^{n}\left(s_{l}-s_{l-1}\right)\left|\sum_{j=l}^{n} w\left(s_{j}\right)\right|^{2} \leq T \sum_{l=1}^{n}\left|\sum_{j=l}^{n}\|w\|_{C_{0}(\mathbb{B})}\right|^{2} \\
= & T\|w\|_{C_{0}(\mathbb{B})}^{2} \sum_{l=1}^{n} l^{2}=\frac{T}{6}\|w\|_{C_{0}(\mathbb{B})}^{2} n(n+1)(2 n+1) \equiv \beta
\end{aligned}
$$

and, for $\alpha \in \mathbb{R}$, we also have

$$
\begin{aligned}
& \int_{\Delta_{n} \times \mathcal{H}^{n}} \int_{C_{0}(\mathbb{B})} \exp \left\{\alpha \sum_{j=1}^{n}\left(w\left(s_{j}\right), x\left(s_{j}\right)\right)^{\sim}\right\} d m_{\mathbb{B}}(x) d\left\|\mu_{F_{n}}\right\|\left(\vec{s}_{n}, \vec{h}_{n}\right) \\
= & \int_{\Delta_{n} \times \mathcal{H}^{n}} \exp \left\{\frac{\alpha^{2}}{2} \sum_{l=1}^{n}\left(s_{l}-s_{l-1}\right)\left|\sum_{j=l}^{n} w\left(s_{j}\right)\right|^{2}\right\} d\left\|\mu_{F_{n}}\right\|\left(\vec{s}_{n}, \vec{h}_{n}\right) \\
\leq & \exp \left\{\frac{\alpha^{2} \beta}{2}\right\}\left\|\mu_{F_{n}}\right\|<\infty
\end{aligned}
$$


FOURIER-FEYNMAN TRANSFORM AND FIRST VARIATION

so that

$$
\begin{aligned}
& \int_{\Delta_{n} \times \mathcal{H}^{n}} \int_{C_{0}(\mathbb{B})}\left|\sum_{l=1}^{n}\left(s_{l}-s_{l-1}\right)\left\langle\sum_{j=l}^{n} h_{j}, \sum_{j=l}^{n} w\left(s_{j}\right)\right\rangle\right| \exp \left\{\alpha \sum _ { j = 1 } ^ { n } \left(w\left(s_{j}\right),\right.\right. \\
& \left.\left.x\left(s_{j}\right)\right)^{\sim}\right\} d m_{\mathbb{B}}(x) d\left\|\mu_{F_{n}}\right\|\left(\vec{s}_{n}, \vec{h}_{n}\right) \\
& <\infty
\end{aligned}
$$

by (17). Now, differentiating both sides of (19) as a function of $t$ for $|t|<\epsilon$ with $\epsilon>0$, arbitrarily, and letting $t=0$, by [9, Theorem 2.27], we have the result.

Remark 2.14 Suppose that the assumptions given as in Lemma 2.13 hold. For $E \in \mathcal{B}\left(\Delta_{n} \times \mathcal{H}^{n}\right)$, let

$$
\mu_{F_{n}}^{w}(E)=\int_{E} i \sum_{l=1}^{n}\left[\left(s_{l}-s_{l-1}\right)\left\langle\sum_{j=l}^{n} h_{j}, \sum_{j=l}^{n} w\left(s_{j}\right)\right\rangle\right] d \mu_{F_{n}}\left(\vec{s}_{n}, \vec{h}_{n}\right)
$$

and let $F_{n}^{w}$ be given by (5) with replacing $\mu_{F_{n}}$ by $\mu_{F_{n}}^{w}$. Then, $F_{n}^{w} \in S_{n, \mathbb{B}}^{\prime \prime}$ and another expression of (18) is given by

$$
\begin{aligned}
& \int_{C_{0}(\mathbb{B})} F_{n}^{w}(x) d m_{\mathbb{B}}(x) \\
= & \int_{C_{0}(\mathbb{B})} \int_{\Delta_{n} \times \mathcal{H}^{n}} F_{\vec{s}_{n}, \vec{h}_{n}}(x) \sum_{j=1}^{n}\left(w\left(s_{j}\right), x\left(s_{j}\right)\right)^{\sim} d \mu_{F_{n}}\left(\vec{s}_{n}, \vec{h}_{n}\right) d m_{\mathbb{B}}(x) .
\end{aligned}
$$

Theorem 2.15 Suppose that the assumptions given as in Lemma 2.13 hold. Then, for $q \in \mathbb{R}-\{0\}$, we have

$$
\begin{aligned}
& \int_{\Delta_{n} \times \mathcal{H}^{n}}\left[\sum_{l=1}^{n}\left(s_{l}-s_{l-1}\right)\left\langle\sum_{j=l}^{n} h_{j}, \sum_{j=l}^{n} w\left(s_{j}\right)\right\rangle\right] \int_{C_{0}(\mathbb{B})}^{a n f_{q}} F_{\vec{s}_{n}, \vec{h}_{n}}(x) \\
& d m_{\mathbb{B}}(x) d \mu_{F_{n}}\left(\vec{s}_{n}, \vec{h}_{n}\right) \\
= & -q \int_{\Delta_{n} \times \mathcal{H}^{n}} \int_{C_{0}(\mathbb{B})}^{a n f_{q}} F_{w, \vec{s}_{n}, \vec{h}_{n}}(x) d m_{\mathbb{B}}(x) d \mu_{F_{n}}\left(\vec{s}_{n}, \vec{h}_{n}\right)
\end{aligned}
$$

with the existences of the Feynman integrals of the both sides of the equality.

Proof. By (16) and (17), for $\lambda>0$, we have

$$
\begin{aligned}
& \frac{i}{\lambda} \int_{\Delta_{n} \times \mathcal{H}^{n}}\left[\sum_{l=1}^{n}\left(s_{l}-s_{l-1}\right)\left\langle\sum_{j=l}^{n} h_{j}, \sum_{j=l}^{n} w\left(s_{j}\right)\right\rangle\right] \int_{C_{0}(\mathbb{B})} F_{\vec{s}_{n}, \vec{h}_{n}}\left(\lambda^{-\frac{1}{2}} x\right) \\
& d m_{\mathbb{B}}(x) d \mu_{F_{n}}\left(\vec{s}_{n}, \vec{h}_{n}\right) \\
= & \int_{\Delta_{n} \times \mathcal{H}^{n}} \int_{C_{0}(\mathbb{B})} F_{w, \vec{s}_{n}, \vec{h}_{n}}\left(\lambda^{-\frac{1}{2}} x\right) d m_{\mathbb{B}}(x) d \mu_{F_{n}}\left(\vec{s}_{n}, \vec{h}_{n}\right)
\end{aligned}
$$


Letting $\lambda \rightarrow-i q$ through $\mathbb{C}_{+}$, we have the result by Morera's and the dominated convergence theorems.

Theorem 2.16 Let $w \in C_{0}(\mathbb{B})$ with $w(s) \in \mathcal{H}$ for all $s \in[0, T]$. Suppose that

$$
\sum_{n=1}^{\infty} \int_{\Delta_{n} \times \mathcal{H}^{n}}\left|\sum_{l=1}^{n}\left(s_{l}-s_{l-1}\right)\left\langle\sum_{j=l}^{n} h_{j}, \sum_{j=l}^{n} w\left(s_{j}\right)\right\rangle\right| d\left\|\mu_{F_{n}}\right\|\left(\vec{s}_{n}, \vec{h}_{n}\right)<\infty
$$

where $\mu_{F_{n}} \in \mathcal{M}_{n}^{\prime \prime}$ for each $n \in \mathbb{N}$ and $\vec{s}_{n}, \vec{h}_{n}$ are given by (2). Then, for $q \in \mathbb{R}-\{0\}$, we have

$$
\begin{aligned}
& \sum_{n=1}^{\infty} \int_{\Delta_{n} \times \mathcal{H}^{n}}\left[\sum_{l=1}^{n}\left(s_{l}-s_{l-1}\right)\left\langle\sum_{j=l}^{n} h_{j}, \sum_{j=l}^{n} w\left(s_{j}\right)\right\rangle\right] \int_{C_{0}(\mathbb{B})}^{a n f_{q}} F_{\vec{s}_{n}, \vec{h}_{n}}(x) \\
& d m_{\mathbb{P}}(x) d \mu_{F_{n}}\left(\vec{s}_{n}, \vec{h}_{n}\right) \\
= & -q \sum_{n=1}^{\infty} \int_{\Delta_{n} \times \mathcal{H}^{n}} \int_{C_{0}(\mathbb{B})}^{a n f_{q}} F_{w, \vec{s}_{n}, \vec{h}_{n}}(x) d m_{\mathbb{B}}(x) d \mu_{F_{n}}\left(\vec{s}_{n}, \vec{h}_{n}\right)
\end{aligned}
$$

with the convergence of the series of both sides of the equality, where $F_{\vec{s}_{n}, \vec{h}_{n}}$, $F_{w, \vec{s}_{n}, \vec{h}_{n}}$ are given by (3), (4), respectively.

Proof. By (20), (21) and the dominated convergence theorem, for $\lambda>0$, we have

$$
\begin{aligned}
& \sum_{n=1}^{\infty} \frac{i}{\lambda} \int_{\Delta_{n} \times \mathcal{H}^{n}}\left[\sum_{l=1}^{n}\left(s_{l}-s_{l-1}\right)\left\langle\sum_{j=l}^{n} h_{j}, \sum_{j=l}^{n} w\left(s_{j}\right)\right\rangle\right] \int_{C_{0}(\mathbb{B})} F_{\vec{s}_{n}, \vec{h}_{n}}\left(\lambda^{-\frac{1}{2}} x\right) \\
& d m_{\mathbb{B}}(x) d \mu_{F_{n}}\left(\vec{s}_{n}, \vec{h}_{n}\right) \\
= & \sum_{n=1}^{\infty} \int_{\Delta_{n} \times \mathcal{H}^{n}} \int_{C_{0}(\mathbb{B})} F_{w, \vec{s}_{n}, \vec{h}_{n}}\left(\lambda^{-\frac{1}{2}} x\right) d m_{\mathbb{B}}(x) d \mu_{F_{n}}\left(\vec{s}_{n}, \vec{h}_{n}\right) .
\end{aligned}
$$

Letting $\lambda \rightarrow-i q$ through $\mathbb{C}_{+}$, we have the result by Theorem 2.15, Morera's and the dominated convergence theorems.

\section{First variation and Fourier-Feynman trans- form of cylinder type functions}

For a given extended real number $p$ with $1<p \leq \infty$, suppose that $p$ and $p^{\prime}$ are related by $\frac{1}{p}+\frac{1}{p^{\prime}}=1$ (possibly $p^{\prime}=1$ if $p=\infty$ ). Let $G_{n}$ and $G$ be measurable functions such that, for each $\gamma>0$,

$$
\lim _{n \rightarrow \infty} \int_{C_{0}(\mathbb{R})}\left|G_{n}(\gamma x)-G(\gamma x)\right|^{p^{\prime}} d m_{\mathbb{B}}(x)=0 .
$$


Then we write

$$
\underset{n \rightarrow \infty}{\lim .}\left(w_{s}^{p^{\prime}}\right)\left(G_{n}\right) \approx G
$$

and call $G$ the scale-invariant limit in the mean of order $p^{\prime}$. A similar definition is understood when $n$ is replaced by a continuously varying parameter.

Now, we define Fourier-Feynman transform of functions over Wiener paths in abstract Wiener space.

Definition 3.1 Let $F$ be defined on $C_{0}(\mathbb{B})$ and for $\lambda \in \mathbb{C}_{+}$let

$$
T_{\lambda}(F)(y)=E^{a n w_{\lambda}}[F(\cdot+y)]
$$

for s-a.e. $y \in C_{0}(\mathbb{B})$ if it exists. For a non-zero real $q$, we define the $L_{1}$ Fourier-Feynman transform $T_{q}^{(1)}(F)$ of $F$ by the formula

$$
T_{q}^{(1)}(F)(y)=E^{a n f_{q}}[F(\cdot+y)]
$$

if it exists for s-a.e. $y \in C_{0}(\mathbb{B})$ and for $1<p \leq \infty$ we define the $L_{p}$ FourierFeynman transform $T_{q}^{(p)}(F)$ of $F$ by the formula

$$
T_{q}^{(p)}(F) \approx \lim _{\lambda \rightarrow-i q}\left(w_{s}^{p^{\prime}}\right)\left(T_{\lambda}(F)\right)
$$

where $\lambda$ approaches to $-i q$ through $\mathbb{C}_{+}$.

Lemma 3.2 Let $F_{\vec{s}_{n}, \vec{h}_{n}}$ be given by (3). Let $q \in \mathbb{R}-\{0\}$ and $1 \leq p \leq \infty$. Then $T_{q}^{(p)}\left(F_{\vec{s}_{n}, \vec{h}_{n}}\right)$ exists and it is given by

$$
T_{q}^{(p)}\left(F_{\vec{s}_{n}, \vec{h}_{n}}\right)(y)=F_{\vec{s}_{n}, \vec{h}_{n}}(y) \exp \left\{-\frac{i}{2 q} \sum_{l=1}^{n}\left(s_{l}-s_{l-1}\right)\left|\sum_{j=l}^{n} h_{j}\right|^{2}\right\}
$$

for s-a.e. $y \in C_{0}(\mathbb{B})$. In particular, if $n=1, h_{1} \equiv h \in \mathcal{H}$ and $s_{1}=u$ with $0<u \leq T$, then (22) is reduced to the following equation

$$
T_{q}^{(p)}\left(F_{u, h}\right)(y)=F_{u, h}(y) \exp \left\{-\frac{i u}{2 q}|h|^{2}\right\}
$$

for s-a.e. $y \in C_{0}(\mathbb{B})$.

Proof. For $\lambda>0$ and s-a.e. $y \in C_{0}(\mathbb{B})$, by Lemma 2.6, we have

$$
\begin{aligned}
T_{\lambda}\left(F_{\vec{s}_{n}, \vec{h}_{n}}\right)(y) & =\int_{C_{0}(\mathbb{R})} F_{\vec{s}_{n}, \vec{h}_{n}}\left(\lambda^{-\frac{1}{2}} x+y\right) d m_{\mathbb{B}}(x) \\
& =F_{\vec{s}_{n}, \vec{h}_{n}}(y) \int_{C_{0}(\mathbb{B})} F_{\vec{s}_{n}, \vec{h}_{n}}\left(\lambda^{-\frac{1}{2}} x\right) d m_{\mathbb{B}}(x) \\
& =F_{\vec{s}_{n}, \vec{h}_{n}}(y) \exp \left\{-\frac{1}{2 \lambda} \sum_{l=1}^{n}\left(s_{l}-s_{l-1}\right)\left|\sum_{j=l}^{n} h_{j}\right|^{2}\right\}
\end{aligned}
$$


and the result holds for $\lambda \in \mathbb{C}_{+}$by Morera's theorem. Let $T_{q}^{(p)}\left(F_{\vec{s}_{n}, \vec{h}_{n}}\right)$ be given by $(22)$. If $p=1$, then $T_{q}^{(1)}\left(F_{\vec{s}_{n}, \vec{h}_{n}}\right)$ is the genuine $L_{1}$ Fourier-Feynman transform of $F_{\vec{s}_{n}, \vec{h}_{n}}$ by the continuity of the exponential function. For $\gamma>0$ and $\frac{1}{p}+\frac{1}{p^{\prime}}=1(1<p \leq \infty)$, we have

$$
\begin{aligned}
& \int_{C_{0}(\mathbb{B})}\left|T_{\lambda}\left(F_{\vec{s}_{n}, \vec{h}_{n}}\right)(\gamma y)-T_{q}^{(p)}\left(F_{\vec{s}_{n}, \vec{h}_{n}}\right)(\gamma y)\right|^{p^{\prime}} d m_{\mathbb{B}}(y) \\
\leq & \int_{C_{0}(\mathbb{P})} \mid \exp \left\{-\frac{1}{2 \lambda} \sum_{l=1}^{n}\left(s_{l}-s_{l-1}\right)\left|\sum_{j=l}^{n} h_{j}\right|^{2}\right\} \\
& -\left.\exp \left\{-\frac{i}{2 q} \sum_{l=1}^{n}\left(s_{l}-s_{l-1}\right)\left|\sum_{j=l}^{n} h_{j}\right|^{2}\right\}\right|^{p^{\prime}} d m_{\mathbb{B}}(y)
\end{aligned}
$$

which tends to 0 as $\lambda$ approaches to $-i q$ through $\mathbb{C}_{+}$by the dominated convergence theorem. Hence, the results follow.

The following theorem follows immediately from Lemmas 2.3 and 3.2.

Theorem 3.3 Suppose that the assumptions given as in Lemma 3.2 hold. Then, for $w \in C_{0}(\mathbb{B})$, we have

$$
\begin{aligned}
& T_{q}^{(p)}\left(\delta_{w} F_{\vec{s}_{n}, \vec{h}_{n}}\right)(y) \\
= & \delta_{w} T_{q}^{(p)}\left(F_{\vec{s}_{n}, \vec{h}_{n}}\right)(y) \\
= & i F_{\vec{s}_{n}, \vec{h}_{n}}(y) \exp \left\{-\frac{i}{2 q} \sum_{l=1}^{n}\left(s_{l}-s_{l-1}\right)\left|\sum_{j=l}^{n} h_{j}\right|^{2}\right\} \sum_{j=1}^{n}\left(h_{j}, w\left(s_{j}\right)\right)^{\sim}
\end{aligned}
$$

for s-a.e. $y \in C_{0}(\mathbb{B})$.

Theorem 3.4 Let $F_{n}, F$ be given by (5), (6), respectively, let $q \in \mathbb{R}-\{0\}$ and $1 \leq p \leq \infty$. Then, both $T_{q}^{(p)}\left(F_{n}\right)(y)$ and $T_{q}^{(p)}(F)(y)$ exist for s-a.e. $y \in$ $C_{0}(\mathbb{B})$ and they are given by

$$
T_{q}^{(p)}\left(F_{n}\right)(y)=\int_{\Delta_{n} \times \mathcal{H}^{n}} T_{q}^{(p)}\left(F_{\vec{s}_{n}, \vec{h}_{n}}\right)(y) d \mu_{F_{n}}\left(\vec{s}_{n}, \vec{h}_{n}\right)
$$

where $T_{q}^{(p)}\left(F_{\vec{s}_{n}, \vec{h}_{n}}\right)$ is given by (22). Moreover, for s-a.e. $y \in C_{0}(\mathbb{B})$, we have

$$
T_{q}^{(p)}(F)(y)=\sum_{n=1}^{\infty} T_{q}^{(p)}\left(F_{n}\right)(y)
$$

where $T_{q}^{(p)}\left(F_{n}\right)$ is given by (23). 
Proof. The results follow from [5, Lemma 3.1 and Theorem 3.5] for $1 \leq p<\infty$. It remains to prove the results for the case $p=\infty$. Let $T_{q}^{(\infty)}\left(F_{n}\right), T_{q}^{(\infty)}(F)$ be given by (23), (24), respectively. By [5, Lemma 3.1 and Theorem 3.5], for $\lambda \in \mathbb{C}_{+}$and $\gamma>0$, we have

$$
\begin{aligned}
& \int_{C_{0}(\mathbb{R})}\left|T_{\lambda}\left(F_{n}\right)(\gamma y)-T_{q}^{(\infty)}\left(F_{n}\right)(\gamma y)\right| d m_{\mathbb{B}}(y) \\
= & \int_{C_{0}(\mathbb{B})} \mid \int_{\Delta_{n} \times \mathcal{H}^{n}} F_{\vec{s}_{n}, \vec{h}_{n}}(\gamma y)\left[\exp \left\{-\frac{1}{2 \lambda} \sum_{l=1}^{n}\left(s_{l}-s_{l-1}\right)\left|\sum_{j=l}^{n} h_{j}\right|^{2}\right\}\right. \\
& \left.-\exp \left\{-\frac{i}{2 q} \sum_{l=1}^{n}\left(s_{l}-s_{l-1}\right)\left|\sum_{j=l}^{n} h_{j}\right|^{2}\right\}\right] d \mu_{F_{n}}\left(\vec{s}_{n}, \vec{h}_{n}\right) \mid d m_{\mathbb{B}}(y) \\
\leq & \int_{\Delta_{n} \times \mathcal{H}^{n}} \mid \exp \left\{-\frac{1}{2 \lambda} \sum_{l=1}^{n}\left(s_{l}-s_{l-1}\right)\left|\sum_{j=l}^{n} h_{j}\right|^{2}\right\} \\
& -\exp \left\{-\frac{i}{2 q} \sum_{l=1}^{n}\left(s_{l}-s_{l-1}\right)\left|\sum_{j=l}^{n} h_{j}\right|^{2}\right\} \mid d\left\|\mu_{F_{n}}\right\|\left(\vec{s}_{n}, \vec{h}_{n}\right)
\end{aligned}
$$

and

$$
\begin{aligned}
& \int_{C_{0}(\mathbb{B})}\left|T_{\lambda}(F)(\gamma y)-T_{q}^{(\infty)}(F)(\gamma y)\right| d m_{\mathbb{B}}(y) \\
\leq & \sum_{n=1}^{\infty} \int_{C_{0}(\mathbb{B})}\left|T_{\lambda}\left(F_{n}\right)(\gamma y)-T_{q}^{(\infty)}\left(F_{n}\right)(\gamma y)\right| d m_{\mathbb{B}}(y) \\
\leq & \sum_{n=1}^{\infty} \int_{\Delta_{n} \times \mathcal{H}^{n}} \mid \exp \left\{-\frac{1}{2 \lambda} \sum_{l=1}^{n}\left(s_{l}-s_{l-1}\right)\left|\sum_{j=l}^{n} h_{j}\right|^{2}\right\} \\
& -\exp \left\{-\frac{i}{2 q} \sum_{l=1}^{n}\left(s_{l}-s_{l-1}\right)\left|\sum_{j=l}^{n} h_{j}\right|^{2}\right\} \mid d\left\|\mu_{F_{n}}\right\|\left(\vec{s}_{n}, \vec{h}_{n}\right) .
\end{aligned}
$$

By the dominated convergence theorem, letting $\lambda \rightarrow-i q$ through $\mathbb{C}_{+}$, the proof is completed.

Remark 3.5 Suppose that the assumptions given as in Theorem 3.4 hold. For $E \in \mathcal{B}\left(\Delta_{n} \times \mathcal{H}^{n}\right)$, let

$$
\mu_{F_{n}}^{q}(E)=\int_{E} \exp \left\{-\frac{i}{2 q} \sum_{l=1}^{n}\left(s_{l}-s_{l-1}\right)\left|\sum_{j=l}^{n} h_{j}\right|^{2}\right\} d \mu_{F_{n}}\left(\vec{s}_{n}, \vec{h}_{n}\right)
$$

and let $F_{n}^{q}, F^{q}$ be given by (5), (6), respectively, with replacing $\mu_{F_{n}}$ by $\mu_{F_{n}}^{q}$. Then $T_{q}^{(p)}\left(F_{n}\right)=F_{n}^{q} \in S_{n, \mathbb{R}}^{\prime \prime}$ and $T_{q}^{(p)}(F)=F^{q} \in S_{\mathbb{B}}^{\prime \prime}$ so that $T_{-q}^{(p)}\left(T_{q}^{(p)}\left(F_{n}\right)\right) \approx F_{n}$ 
and $T_{-q}^{(p)}\left(T_{q}^{(p)}(F)\right) \approx F$, that is, $T_{-q}^{(p)}$ is the inverse transform of $T_{q}^{(p)}$ by Theorem 3.4. Thus we have the following theorem.

Theorem 3.6 Suppose that the assumptions given as in Theorem 3.4 hold. Let $w \in C_{0}(\mathbb{B})$ and suppose that (10) holds. Then, for s-a.e. $y \in C_{0}(\mathbb{B})$, we have

$$
T_{q}^{(p)}\left(\delta_{w} F_{n}\right)(y)=\delta_{w}\left(T_{q}^{(p)}\left(F_{n}\right)\right)(y)
$$

and

$$
T_{q}^{(p)}\left(\delta_{w} F\right)(y)=\delta_{w}\left(T_{q}^{(p)}(F)\right)(y)
$$

with the existences of both sides of the equalities.

Proof. For any Borel subset $E$ of $\Delta_{n} \times \mathcal{H}^{n}$, let

$$
\mu_{F_{n}}^{w}(E)=\int_{E} i \sum_{j=1}^{n}\left(h_{j}, w\left(s_{j}\right)\right)^{\sim} d \mu_{F_{n}}\left(\vec{s}_{n}, \vec{h}_{n}\right)
$$

where $\vec{s}_{n}$ and $\vec{h}_{n}$ are given by (2). Then we have $\mu_{F_{n}}^{w} \in \mathcal{M}_{n}^{\prime \prime}$ so that $\delta_{w} F_{n} \in S_{n, \mathbb{B}}^{\prime \prime}$ by Theorem 2.4. Thus, for $s$-a.e. $y \in C_{0}(\mathbb{B})$, we have

$$
\begin{aligned}
& T_{q}^{(p)}\left(\delta_{w} F_{n}\right)(y) \\
= & \int_{\Delta_{n} \times \mathcal{H}^{n}} T_{q}^{(p)}\left(F_{\vec{s}_{n}, \vec{h}_{n}}\right)(y) d \mu_{F_{n}}^{w}\left(\vec{s}_{n}, \vec{h}_{n}\right) \\
= & \int_{\Delta_{n} \times \mathcal{H}^{n}} T_{q}^{(p)}\left(\delta_{w} F_{\vec{s}_{n}, \vec{h}_{n}}\right)(y) d \mu_{F_{n}}\left(\vec{s}_{n}, \vec{h}_{n}\right) \\
= & \int_{\Delta_{n} \times \mathcal{H}^{n}} \delta_{w} T_{q}^{(p)}\left(F_{\vec{s}_{n}, \vec{h}_{n}}\right)(y) d \mu_{F_{n}}\left(\vec{s}_{n}, \vec{h}_{n}\right) \\
= & \int_{\Delta_{n} \times \mathcal{H}^{n}} \delta_{w} F_{\vec{s}_{n}, \vec{h}_{n}}(y) d \mu_{F_{n}}^{q}\left(\vec{s}_{n}, \vec{h}_{n}\right) \\
= & \delta_{w}\left(T_{q}^{(p)}\left(F_{n}\right)\right)(y)
\end{aligned}
$$

by (9), (23), (28) and Theorem 3.3, where $\mu_{F_{n}}^{q}$ is given by (25). This completes the proof of (26). (27) follows immediately from (26).

Remark 3.7 For the result in (26), the condition (10) can be replaced by the condition (8). 
FOURIER-FEYNMAN TRANSFORM AND FIRST VARIATION

Theorem 3.8 Suppose that the assumptions given as in Theorem 2.11 hold. Then, for $1 \leq p \leq \infty$, we have

$$
\begin{aligned}
& T_{q}^{(p)}\left(F_{w, \vec{s}_{n}, \vec{h}_{n}}\right)(y) \\
= & -\frac{1}{q}\left[\sum_{l=1}^{n}\left(s_{l}-s_{l-1}\right)\left\langle\sum_{j=l}^{n} h_{j}, \sum_{j=l}^{n} w\left(s_{j}\right)\right\rangle\right] T_{q}^{(p)}\left(F_{\vec{s}_{n}, \vec{h}_{n}}\right)(y) \\
+ & {\left[\sum_{j=1}^{n}\left(w\left(s_{j}\right), y\left(s_{j}\right)\right)^{\sim}\right] T_{q}^{(p)}\left(F_{\vec{s}_{n}, \vec{h}_{n}}\right)(y) }
\end{aligned}
$$

for s-a.e. $y \in C_{0}(\mathbb{B})$.

Proof. For $\lambda>0$, multiplying $F_{\vec{s}_{n}, \vec{h}_{n}}(y)$ to the both sides of (16), we have

$$
\begin{aligned}
& \frac{i}{\lambda}\left[\sum_{l=1}^{n}\left(s_{l}-s_{l-1}\right)\left\langle\sum_{j=l}^{n} h_{j}, \sum_{j=l}^{n} w\left(s_{j}\right)\right\rangle\right] \int_{C_{0}(\mathbb{B})} F_{\vec{s}_{n}, \vec{h}_{n}}\left(\lambda^{-\frac{1}{2}} x+y\right) d m_{\mathbb{B}}(x) \\
= & \int_{C_{0}(\mathbb{B})} F_{w, \vec{s}_{n}, \vec{h}_{n}}\left(\lambda^{-\frac{1}{2}} x+y\right) d m_{\mathbb{B}}(x)-\left[\sum_{j=1}^{n}\left(w\left(s_{j}\right), y\left(s_{j}\right)\right)^{\sim}\right] \\
& \times \int_{C_{0}(\mathbb{B})} F_{\vec{s}_{n}, \vec{h}_{n}}\left(\lambda^{-\frac{1}{2}} x+y\right) d m_{\mathbb{B}}(x)
\end{aligned}
$$

for $s$-a.e. $y \in C_{0}(\mathbb{B})$. Now, $T_{q}^{(p)}\left(F_{\vec{s}_{n}, \vec{h}_{n}}\right)$ exists by Lemma 3.2 and letting $\lambda \rightarrow-i q$ through $\mathbb{C}_{+}$, we have the result.

Remark 3.9 Let $1 \leq p \leq \infty$. Under the assumptions given as in Remark 2.12, we have

$$
\begin{aligned}
& T_{q}^{(p)}\left(F_{w, u, h}\right)(y) \\
= & \frac{i u}{q} T_{q}^{(p)}\left(\delta_{w} F_{u, h}\right)(y)+(w(u), y(u))^{\sim} T_{q}^{(p)}\left(F_{u, h}\right)(y)
\end{aligned}
$$

for s-a.e. $y \in C_{0}(\mathbb{B})$.

Theorem 3.10 Suppose that the assumptions given as in Theorem 2.15 hold. Then, for $1 \leq p \leq \infty$, we have

$$
\begin{aligned}
& \int_{\Delta_{n} \times \mathcal{H}^{n}} T_{q}^{(p)}\left(F_{w, \vec{s}_{n}, \vec{h}_{n}}\right)(y) d \mu_{F_{n}}\left(\vec{s}_{n}, \vec{h}_{n}\right) \\
= & -\frac{1}{q} \int_{\Delta_{n} \times \mathcal{H}^{n}}\left[\sum_{l=1}^{n}\left(s_{l}-s_{l-1}\right)\left\langle\sum_{j=l}^{n} h_{j}, \sum_{j=l}^{n} w\left(s_{j}\right)\right\rangle\right] T_{q}^{(p)}\left(F_{\vec{s}_{n}, \vec{h}_{n}}\right)(y) d \mu_{F_{n}}\left(\vec{s}_{n}, \vec{h}_{n}\right) \\
& +\int_{\Delta_{n} \times \mathcal{H}^{n}}\left[\sum_{j=1}^{n}\left(w\left(s_{j}\right), y\left(s_{j}\right)\right)^{\sim}\right] T_{q}^{(p)}\left(F_{\vec{s}_{n}, \vec{h}_{n}}\right)(y) d \mu_{F_{n}}\left(\vec{s}_{n}, \vec{h}_{n}\right)
\end{aligned}
$$

for s-a.e. $y \in C_{0}(\mathbb{B})$. 
Proof. By (17) and (22), the first integral of the right hand side of the equality exists. Using the process given as in the proof of Lemma 2.13, by (22), we have

$$
\int_{\Delta_{n} \times \mathcal{H}^{n}} \int_{C_{0}(\mathbb{B})}\left|T_{q}^{(p)}\left(F_{\vec{s}_{n}, \vec{h}_{n}}\right)(y) \sum_{j=1}^{n}\left(w\left(s_{j}\right), y\left(s_{j}\right)\right)\right| d m_{\mathbb{B}}(y) d\left\|\mu_{F_{n}}\right\|\left(\vec{s}_{n}, \vec{h}_{n}\right)<\infty
$$

so that the second integral of the right hand side of the equality exists for $s$-a.e. $y$ in $C_{0}(\mathbb{B})$. Now, integrating both sides of the equation given as in Theorem 3.8 with respect to $\mu_{F_{n}}$, we have the result.

Now, by (21), (22) and Theorem 3.10, we have the following theorem.

Theorem 3.11 Suppose that the assumptions given as in Theorem 2.16 hold. Let $1 \leq p \leq \infty$ and suppose that $\sum_{n=1}^{\infty} \int_{\Delta_{n} \times \mathcal{H}^{n}} T_{q}^{(p)}\left(F_{w, \vec{s}_{n}, \vec{h}_{n}}\right)(y) d \mu_{F_{n}}\left(\vec{s}_{n}, \vec{h}_{n}\right)$ exists for s-a.e. $y \in C_{0}(\mathbb{B})$. Then we have

$$
\begin{aligned}
& \sum_{n=1}^{\infty} \int_{\Delta_{n} \times \mathcal{H}^{n}} T_{q}^{(p)}\left(F_{w, \vec{s}_{n}, \vec{h}_{n}}\right)(y) d \mu_{F_{n}}\left(\vec{s}_{n}, \vec{h}_{n}\right) \\
= & -\frac{1}{q} \sum_{n=1}^{\infty} \int_{\Delta_{n} \times \mathcal{H}^{n}}\left[\sum_{l=1}^{n}\left(s_{l}-s_{l-1}\right)\left\langle\sum_{j=l}^{n} h_{j}, \sum_{j=l}^{n} w\left(s_{j}\right)\right\rangle\right] T_{q}^{(p)}\left(F_{\vec{s}_{n}, \vec{h}_{n}}\right)(y) \\
& d \mu_{F_{n}}\left(\vec{s}_{n}, \vec{h}_{n}\right)+\sum_{n=1}^{\infty} \int_{\Delta_{n} \times \mathcal{H}^{n}}\left[\sum_{j=1}^{n}\left(w\left(s_{j}\right), y\left(s_{j}\right)\right)^{\sim}\right] T_{q}^{(p)}\left(F_{\vec{s}_{n}, \vec{h}_{n}}\right)(y) d \mu_{F_{n}}\left(\vec{s}_{n}, \vec{h}_{n}\right)
\end{aligned}
$$

for s-a.e. $y \in C_{0}(\mathbb{B})$.

\section{Transformation of cylinder type functions with linear factors}

Let $0<u \leq T$ be fixed, but arbitrarily, let $F_{0}$ be defined on $C_{0}(\mathbb{B})$ and for any given fixed $n \in \mathbb{N}$ let

$$
F_{j}(x)=F_{0}(x) \prod_{l=1}^{j}\left(w_{l}(u), x(u)\right)^{\sim}
$$

for $s$-a.e. $x \in C_{0}(\mathbb{B})$ where $w_{l} \in C_{0}(\mathbb{B})$ with $w_{l}(u) \in \mathcal{H}$ for $l=1, \cdots, n$.

Our first theorem gives a recurrence relation in which we express the transform of $F_{j}$ in terms of the transforms and variation of $F_{j-1}$. The proof follows immediately from the definition of Fourier-Feynman transform([8]). 
Theorem 4.1 For $1 \leq p \leq \infty$, for a non-zero real $q$ and for $j=1, \cdots, n$, assume that both $T_{q}^{(p)}\left(\delta_{w_{j}} F_{j-1}\right)(y)$ and $T_{q}^{(p)}\left(F_{j-1}\right)(y)$ can be expressible as analytic Feynman integrals for $s$-a.e. $y \in C_{0}(\mathbb{B})$. Moreover, $F_{j-1}(\cdot+y)$ satisfies the equation given as in Remark 2.12 with replacing $w, F_{u, h}$ by $w_{j}, F_{j-1}(\cdot+y)$, respectively. Then $T_{q}^{(p)}\left(F_{j}\right)(y)$ exists for s-a.e. $y \in C_{0}(\mathbb{B})$ and it is given by the recurrence relation

$$
T_{q}^{(p)}\left(F_{j}\right)(y)=\frac{i u}{q} T_{q}^{(p)}\left(\delta_{w_{j}} F_{j-1}\right)(y)+\left(w_{j}(u), y(u)\right)^{\sim} T_{q}^{(p)}\left(F_{j-1}\right)(y) .
$$

The following theorem is an immediate result of Theorem 4.1([8]).

Theorem 4.2 For some $\epsilon>0$, the assumptions given as in Theorem 4.1 hold with replacing $F_{j-1}$ by $F_{j-1}\left(\cdot+t w_{j+1}\right)$ for $|t|<\epsilon$ and $j=1, \cdots, n-1$. Moreover, assume that both

$$
T_{q}^{(p)}\left(\delta_{w_{j+1}} F_{j}\right)(y)=\delta_{w_{j+1}}\left(T_{q}^{(p)}\left(F_{j}\right)\right)(y)
$$

and

$$
T_{q}^{(p)}\left(\delta_{w_{j+1}} F_{j-1}\right)(y)=\delta_{w_{j+1}}\left(T_{q}^{(p)}\left(F_{j-1}\right)\right)(y)
$$

exist for s-a.e. $y \in C_{0}(\mathbb{B})$. Then, for s-a.e. $y \in C_{0}(\mathbb{B})$, we have the recurrence relation

$$
\begin{aligned}
T_{q}^{(p)}\left(\delta_{w_{j+1}} F_{j}\right)(y)= & \frac{i u}{q} \delta_{w_{j+1}}\left(T_{q}^{(p)}\left(\delta_{w_{j}} F_{j-1}\right)\right)(y)+\left\langle w_{j}(u), w_{j+1}(u)\right\rangle \\
& \times T_{q}^{(p)}\left(F_{j-1}\right)(y)+\left(w_{j}(u), y(u)\right)^{\sim} T_{q}^{(p)}\left(\delta_{w_{j+1}} F_{j-1}\right)(y) .
\end{aligned}
$$

Now, let $h \in \mathcal{H}$ and $F_{0}$ be given by

$$
F_{0}(x)=\exp \left\{i(h, x(u))^{\sim}\right\}
$$

for $s$-a.e. $x \in C_{0}(\mathbb{B})$. Let $F_{n}$ be given by $(29)$. Then we have

$$
T_{q}^{(p)}\left(F_{1}\right)(y)=\frac{i u}{q} \delta_{w_{1}}\left(T_{q}^{(p)}\left(F_{0}\right)\right)(y)+\left(w_{1}(u), y(u)\right)^{\sim} T_{q}^{(p)}\left(F_{0}\right)(y)
$$

for $s$-a.e. $y \in C_{0}(\mathbb{B})$, by $(15)$, Remarks 2.12, 3.9 and Theorem 3.3. Also, we have

$$
\delta_{w_{1}}\left(T_{q}^{(p)}\left(F_{0}\right)\right)(y)=i\left\langle h, w_{1}(u)\right\rangle \exp \left\{-\frac{i u}{2 q}|h|^{2}\right\} F_{0}(y)
$$

and hence

$$
\delta_{w_{2}}\left(\delta_{w_{1}}\left(T_{q}^{(p)}\left(F_{0}\right)\right)\right)(y)=-\left\langle h, w_{1}(u)\right\rangle\left\langle h, w_{2}(u)\right\rangle \exp \left\{-\frac{i u}{2 q}|h|^{2}\right\} F_{0}(y)
$$


for $s$-a.e. $y \in C_{0}(\mathbb{B})$. Thus, by Theorem 4.2 , we have

$$
\begin{aligned}
T_{q}^{(p)}\left(\delta_{w_{2}} F_{1}\right)(y)= & \frac{i u}{q} \delta_{w_{1}}\left(T_{q}^{(p)}\left(w_{2} F_{0}\right)\right)(y)+\left\langle w_{1}(u), w_{2}(u)\right\rangle T_{q}^{(p)}\left(F_{0}\right)(y) \\
& +\left(w_{1}(u), y(u)\right)^{\sim} T_{q}^{(p)}\left(\delta_{w_{2}} F_{0}\right)(y) \\
= & \delta_{w_{2}}\left(T_{q}^{(p)}\left(F_{1}\right)\right)(y)
\end{aligned}
$$

for $s$-a.e. $y \in C_{0}(\mathbb{B})$. By Theorem 4.1 with $j=2$, we see that

$$
T_{q}^{(p)}\left(F_{2}\right)(y)=\frac{i u}{q} \delta_{w_{2}}\left(T_{q}^{(p)}\left(F_{1}\right)\right)(y)+\left(w_{2}(u), y(u)\right)^{\sim} T_{q}^{(p)}\left(F_{1}\right)(y)
$$

for $s$-a.e. $y \in C_{0}(\mathbb{B})$. Continuing this process, we have

$$
\delta_{w_{n}}\left(\cdots \delta_{w_{1}}\left(T_{q}^{(p)}\left(F_{0}\right)\right) \cdots\right)(y)=\left(\prod_{l=1}^{n} i\left\langle h, w_{l}(u)\right\rangle\right) \exp \left\{-\frac{i u}{2 q}|h|^{2}\right\} F_{0}(y)
$$

for $s$-a.e. $y \in C_{0}(\mathbb{B})$. Hence $\delta_{w_{n}}\left(T_{q}^{(p)}\left(F_{n-1}\right)\right)(y)$ exists for $s$-a.e. $y \in C_{0}(\mathbb{B})$ so that we obtain, by Theorem 4.1,

$$
T_{q}^{(p)}\left(F_{n}\right)(y)=\frac{i u}{q} \delta_{w_{n}}\left(T_{q}^{(p)}\left(F_{n-1}\right)\right)(y)+\left(w_{n}(u), y(u)\right)^{\sim} T_{q}^{(p)}\left(F_{n-1}\right)(y)
$$

for $s$-a.e. $y \in C_{0}(\mathbb{B})$. Thus we have the following theorem.

Theorem 4.3 Under the above assumptions, for $k=1, \cdots, n$, we have

$$
\begin{aligned}
T_{q}^{(p)}\left(F_{k}\right)(y)= & \frac{i u}{q} \sum_{j=0}^{k-1}\left[\delta_{w_{j+1}}\left(T_{q}^{(p)}\left(F_{j}\right)\right)(y)\left(\prod_{l=j+2}^{k}\left(w_{l}(u), y(u)\right)^{\sim}\right)\right] \\
& +T_{q}^{(p)}\left(F_{0}\right)(y)\left(\prod_{l=1}^{k}\left(w_{l}(u), y(u)\right)^{\sim}\right)
\end{aligned}
$$

for s-a.e. $y \in C_{0}(\mathbb{B})$.

\section{References}

[1] M.D. Brue, A functional transform for Feynman integrals similar to the Fourier transform, thesis, Univ. of Minnesota, Minneapolis, 1972.

[2] R.H. Cameron, The first variation of an indefinite Wiener intergal, Proc. Amer. Math. Soc., 2 (1951), 914-924.

[3] R.H. Cameron and D.A. Storvick, An $L_{2}$ analytic Fourier-Feynman transform, Michigan Math. J., 23 (1976), 1-30. 
[4] R.H. Cameron and D.A. Storvick, Some Banach algebras of analytic Feynman integrable functionals, an analytic functions, Kozubnik, Lecture Notes in Math., 798 (1980), 18-27.

[5] K.S. Chang, D.H. Cho, T.S. Song and I. Yoo, Evaluation formulas for Fourier-Feynman transform over paths in abstract Wiener space, J. Interdisciplinary Math., 5 (2002), no.2, 143-164.

[6] K.S. Chang, T.S. Song and I. Yoo, Analytic Fourier-Feynman transform and first variation on abstract Wiener space, J. Korean Math. Soc., 38 (2001), no.2, 485-501.

[7] D.H. Cho, Conditional analytic Feynman integral over product space of Wiener paths in abstract Wiener space, Rocky Mount. J. Math., (2003) submitted.

[8] D.H. Cho, Conditional first variation over Wiener paths in abstract Wiener space, J. Korean Math. Soc., (2004), to appear.

[9] G.B. Folland, Real analysis, John Wiley \& Sons, 1984.

[10] G.W. Johnson and D.L. Skoug, An $L_{p}$ analytic Fourier-Feynman transform, Michigan Math. J., 26 (1979), 103-127.

[11] G. Kallianpur and C. Bromley, Generalized Feynman integrals using analytic continuation in several complex variables, stochastic analysis and applications, Dekker, N.Y., 1984.

[12] J. Kuelbs, Abstract Wiener measure and applications to analysis, Pacific J. Math., 31 (1969), no.2, 433-450.

[13] H.H. Kuo, Gaussian measures in Banach spaces, Lecture Notes in Mathematics 463, Springer-Verlag, 1975.

[14] C. Park, D.L. Skoug and D.A. Storvick, Fourier-Feynman transforms and the first variation, Rendiconti Del Circolo Matematico Di Palermo Serie II, Tomo XLVII, 2 (1998), 277-292.

[15] K.S. Ryu, The Wiener integral over paths in abstract Wiener space, J. Korean Math. Soc., 29 (1992), no.2, 317-331.

[16] I. Yoo, The analytic Feynman integral over paths in abstract Wiener space, Comm. Korean Math. Soc., 10 (1995), no.1, 93-107.

\section{Received: August 1, 2004}

